\title{
Early prediction of recurrent Cushing disease after surgery
}

A coupled dexamethasone-desmopressin test (CDDT) is superior to previously suggested methods for prediction of postsurgical recurrence of Cushing disease, according to a French study.

Cushing disease recurs in about $25 \%$ of patients who undergo trans-sphenoidal surgery. Desmopressin stimulation tests can predict postsurgical recurrence; however, this test has only $80 \%$ sensitivity and specificity. Castinetti and colleagues assessed whether combining this method with a low-dose dexamethasone suppression test could improve these parameters.

In 38 patients who were treated with trans-sphenoidal surgery for Cushing disease, a CDDT (1 mg dexamethasone followed by $10 \mu \mathrm{g}$ intravenous desmopressin) was performed 3-6 months after surgery and yearly thereafter. The investigators evaluated the maximal increase in the levels of adrenocorticotropic hormone and cortisol after the desmopressin injection relative to baseline levels, which were determined after a low-dose dexamethasone suppression test.

Positive test results (a relative increase of $\geq 50 \%$ in adrenocorticotropic hormone and cortisol levels) were obtained in all 10 patients with recurrence and in three patients who remained in remission during the follow-up period (mean 60 months). Thus, the CDDT had $100 \%$ sensitivity and $89 \%$ specificity. Notably, in eight of the 10 patients with recurrence, the test predicted this outcome earlier than other markers, such as $24 \mathrm{~h}$ urinary free cortisol levels.

Julianna Vig

Original article Castinetti, F. et al. A combined dexamethasone desmopressin test as an early marker of post-surgical recurrence in Cushing's disease. J. Clin. Endocrinol. Metab. doi:10.1210/jc.2008-2234 